\title{
Fisioterapia em recém-nascidos com persistência do canal arterial e complicações pulmonares
}

\author{
Chest physical therapy in newborn infants with patent ductus arteriosus and pulmonary complications
}

Ivete Furtado Ribeiro', Ana Paula L. de Melo², Josy Davidson ${ }^{3}$

\section{RESUMO}

Objetivos: Revisar as complicações pulmonares e intervenções fisioterapêuticas disponíveis para recém-nascidos com persistência do canal arterial (PCA).

Fontes de dados: Artigos publicados nas fontes de dados Medline e Lilacs, além de capítulos de livros nos idiomas português e inglês, entre 1992 e 2006 . As palavras-chave utilizadas para busca foram: "canal arterial", "prematuridade", "síndrome do desconforto respiratório", "complicações" e "pulmão".

Síntese dos dados: A PCA é uma intercorrência freqüente em recém-nascidos pré-termos, que pode se associar a complicações pulmonares, tais como atelectasias, infecções pulmonares e falha na extubação, aumentando o tempo de ventilação mecânica e de internação na Unidade de Terapia Intensiva Neonatal. Os recursos fisioterapêuticos visam melhorar as condições pulmonares, propiciando o aumento da complacência pulmonar e a diminuição da resistência de vias aéreas, além de otimizar o mecanismo de depuração mucociliar e, dessa forma, reduzir o trabalho respiratório.

Conclusões: A fisioterapia respiratória contribui para melhorar a evolução dos neonatos com complicações pulmonares por persistência de canal arterial, permitindo otimizar a mecânica respiratória, além de melhorar a oxigenação e a relação ventilação/perfusão.

Palavras-chave: prematuro; persistência do conduto arterioso; modalidades de Fisioterapia; pulmão.

\section{ABSTRACT}

Objective: To review the pulmonary complications and the available chest physical therapy interventions for neonates with patent ductus arteriosus (PDA).

Data sources: Studies were retrieved from Medline and Lilacs and textbooks in Portuguese and in English, from 1992 to 2006. The following key-words were searched: "ductus arteriosus", "prematurity", "respiratory distress syndrome", "complications", "lung".

Data synthesis: PDA is frequently observed among preterm infants and can be associated with pulmonary complications, such as atelectasis, infections, extubation failure, increased mechanical ventilation dependency and longer length of hospital stay. Studies show that chest physical therapy may improve pulmonary function, increase lung compliance, reduce airway resistance, optimize bronchial toilet and, as a result, decrease the work of breathing.

Conclusions: Chest physical therapy contributes to improve clinical outcome of neonates with pulmonary complications due to patent ductus arteriosus, allowing them to optimize respiratory mechanics, blood oxygenation and ventilation/perfusion ratio.

Key-words: infant, premature; ductus arteriosus, patent; physical therapy modalities; lung.
${ }^{1}$ Especializanda do curso de Fisioterapia Respiratória Pediátrica e Neonatal da Escola Paulista de Medicina da Universidade Federal de São Paulo (Unifesp-EPM), São Paulo, SP, Brasil

2Pós-graduanda em nível de mestrado pelo Programa de Pós-Graduação em Pediatria e Ciências Aplicadas à Pediatria do Departamento de Pediatria da Unifesp-EPM e supervisora do curso de Fisioterapia Respiratória Pediátrica e Neonatal da Unifesp-EPM, São Paulo, SP, Brasil

${ }^{3}$ Pós-graduanda em nível de doutorado pelo Programa de Pós-Graduação em Pediatria e Ciências Aplicadas à Pediatria do Departamento de Pediatria da Unifesp-EPM e supervisora do curso de Fisioterapia Respiratória Pediátrica e Neonatal da Unifesp-EPM, São Paulo, SP, Brasil
Endereço para correspondência:

Ivete Furtado Ribeiro

Avenida Senador Lemos, 1.027 - Umarizal

CEP 66050-000 - Belém/PA

E-mail: ivbeiro@yahoo.com.br

Recebido em: 17/9/2007

Aprovado em: 20/12/2007 


\section{Introdução}

Os avanços na assistência neonatal a partir da década de 1960 contribuíram para aumentar a sobrevida de recém-nascidos pré-termos (RNPT) criticamente doentes. Entretanto, as afecções cardíacas e pulmonares ainda constituem causas importantes de morbimortalidade no período neonatal, prolongando o tempo de internação hospitalar e limitando o prognóstico de tais pacientes ${ }^{(1)}$.

Nesse contexto, pode-se citar a persistência do canal arterial (PCA), que se associa com freqüência à prematuridade e corresponde a 5 a $10 \%$ de todas as cardiopatias, acometendo cerca de $40 \%$ dos prematuros com idade gestacional entre 25 e 27 semanas $^{(1-3)}$.

Além do comprometimento cardíaco, a PCA pode cursar com complicações pulmonares, como atelectasias, infecções pulmonares e falha na retirada da ventilação mecânica, sobretudo, naqueles submetidos à correção cirúrgica da afecção ${ }^{(4,5)}$. Devido a tais intercorrências, a fisioterapia respiratória pode ter importante função na remoção das secreções brônquicas, na otimização da mecânica respiratória, bem como na melhora da oxigenação e da ventilação de recém-nascidos com $\mathrm{PCA}^{(6)}$.

Nesta revisão, serão abordadas as principais repercussões pulmonares da PCA e o papel da fisioterapia respiratória em prematuros, independentemente da correção cirúrgica.

\section{Fisiologia intra-uterina da circulação sistêmica e pulmonar}

Durante a vida intra-uterina, os pulmões não oxigenam o sangue, uma vez que as trocas gasosas ocorrem na placenta. Uma pequena parte do sangue oxigenado na placenta é desviada para o fígado, por meio do ducto venoso, e a outra parte dirige-se para o átrio direito, pela veia cava inferior, de onde é preferencialmente direcionada para o átrio esquerdo pelo forame oval. Do átrio esquerdo, o sangue oxigenado atinge o ventrículo esquerdo e, deste, a aorta. O sangue pré-ductal com maior conteúdo de oxigênio irriga o cérebro, o coração e as adrenais. O sangue pós-ductal, que apresenta menor quantidade de oxigênio porque se mistura com o sangue proveniente do ventrículo direito através do canal arterial, é responsável por irrigar os demais órgãos.

O sangue venoso de drenagem do cérebro dirige-se para a veia cava superior e se mistura com o sangue da veia cava inferior, oriundo da drenagem de membros inferiores e vísceras abominais. Esse sangue, ao atingir o átrio direito, dirige-se preferencialmente ao ventrículo direito e, devido à alta resistência vascular pulmonar e à baixa resistência periférica, apenas 7 a 10\% dele segue para os pulmões através das artérias pulmonares, sendo a maior parte desviada para a aorta por meio do canal arterial, misturando-se ao sangue com maior conteúdo de oxigênio que provém do ventrículo esquerdo. Depois de irrigar os órgãos periféricos, o sangue retorna à placenta para ser novamente oxigenado ${ }^{(1,7)}$

Dessa forma, a manutenção das comunicações entre as circulações pulmonar e sistêmica é fundamental para a sobrevivência fetal e ocorre pela alta resistência vascular pulmonar associada às baixas concentrações de oxigênio e pela grande quantidade de prostaglandinas circulantes, além da baixa resistência sistêmica ${ }^{(1,3)}$.

\section{Transição da circulação sistêmica e pulmonar ao nascimento}

Imediatamente após o nascimento, observa-se a mudança da circulação fetal para neonatal, com redução da resistência vascular pulmonar associada ao aumento do fluxo sanguíneo pulmonar e diminuição da pressão na artéria pulmonar. Além disso, a queda da concentração de prostaglandinas e a retirada da placenta determinam a constrição da camada muscular média das artérias, elevando a pressão arterial sistêmica. Dessa forma, a comunicação entre a aorta e a artéria pulmonar tende ao fechamento, que é potencializado pela rápida contração do canal em resposta à elevação da concentração de oxigênio ${ }^{(1-3)}$.

Já o fechamento do canal arterial ao nascimento ocorre em duas etapas: o fechamento funcional, que ocorre nos primeiros quatro dias de vida e decorre do papel constritor do oxigênio que, com a oxidação do citocromo $A_{3}$, promove a produção de ATP e a contração do canal arterial ${ }^{(1,3-5)}$. O fechamento anatômico segue o fechamento funcional do canal arterial e se deve à isquemia hipóxica profunda na parede muscular do canal, com degeneração da parede, necrose e migração das células musculares, com formação de cistos e proliferação da camada íntima, havendo oclusão gradual do lúmen vascular. Com isso, o canal se transforma em ligamento arterioso ${ }^{(1,3,7)}$.

Entretanto, a resposta à pressão arterial de oxigênio ocorre proporcionalmente ao aumento da idade gestacional, razão pela qual os prematuros são mais propensos a manter a patência do canal arterial ${ }^{(4,7,8)}$. Sabe-se que, com 28 semanas de gestação, não se observa o efeito constritor do $\mathrm{O}_{2}$, nem mesmo com concentrações próximas a $100 \%$. Já nos nascidos a termo, mesmo em ar ambiente, a constrição do canal é evidenciada com o fechamento completo até o quarto dia 
de $\operatorname{vida}^{(7)}$. Além disso, nos prematuros, o fechamento pode ser retardado, sobretudo na presença de asfixia perinatal, síndrome do desconforto respiratório, ventilação mecânica e hipervolemia ${ }^{(5,9)}$. Quando o diâmetro do canal é grande, as chances de fechamento espontâneo são diminutas. Canais menores que $1,7 \mathrm{~mm}$ provavelmente fecharão espontaneamente, enquanto aqueles maiores que $2,2 \mathrm{~mm}$ não terão fechamento espontâneo ${ }^{(3)}$.

\section{Fisiopatologia da PCA no período neonatal}

Com a patência do canal após o nascimento, há presença de shunt esquerdo-direito secundário à inversão do padrão circulatório. Esse shunt depende não só do diâmetro e do tônus do canal, mas também da resistência vascular pulmonar e sistêmica e do desempenho do ventrículo esquerdo, podendo levar a repercussões cardiopulmonares $s^{(1,2,5,10)}$

$\mathrm{Na}$ fase inicial (fase compensada), o neonato consegue manter o fluxo sistêmico à custa da elevação do débito cardíaco, decorrente do aumento da contratilidade miocárdica e da freqüência cardíaca. No entanto, dado o limitado desempenho miocárdico, instala-se a fase descompensada, caracterizada por insuficiência cardíaca e diminuição do débito cardíaco ${ }^{(1,7,10)}$.

É durante a fase de descompensação que se instalam as complicações respiratórias decorrentes do edema pulmonar propiciado pelo shunt E-D, agravado por maior permeabilidade dos capilares pulmonares, menor desenvolvimento dos linfáticos pulmonares e maior imaturidade da membrana alveolar, característicos do prematuro. Como conseqüência, há congestão pulmonar, piora da complacência e da resistência pulmonar, gerando mudanças na mecânica pulmonar e alteração na relação ventilação-perfusão, com hipóxia, hipercapnia e acidose mista ${ }^{(1,3,5,7,10)}$.

\section{Quadro clínico e diagnóstico da PCA}

O diagnóstico da PCA pode ser realizado por sinais clínicos, análise dos gases arteriais, radiografia de tórax, eletrocardiograma e ecocardiograma ${ }^{(1,3,4,7,11-14)}$.

As manifestações clínicas dependem da magnitude do shunt E-D e, portanto, das repercussões cardiocirculatórias, pulmonares e sistêmicas. Ademais, o quadro clínico varia com a idade gestacional do recém-nascido ${ }^{(1,3,4,6,9,11,13)}$ (Quadro 1). Outras manifestações decorrem da diminuição do fluxo sanguíneo sistêmico, como diminuição do débito urinário, distensão abdominal com enterocolite necrosante e hemorragia peri- e/ou intraventicular ${ }^{(1,4,9)}$.

Nos prematuros com peso de nascimento inferior a 1.000 ou $1.500 \mathrm{~g}$, as manifestações clínicas podem aparecer na fase de melhora do quadro da síndrome do desconforto respiratório, evidenciando-se, então, o shunt E-D. Provavelmente, nesses casos, o canal já estava aberto, porém, a resistência vascular pulmonar impedia a ocorrência do shunt na fase inicial da doença pulmonar ${ }^{(1,5,9,13)}$.

Quanto às alterações nos gases arteriais, inicialmente observa-se acidose respiratória, causada pelo edema, e comprometimento da mecânica pulmonar. Com o progredir do quadro, associa-se a hipoxemia que, juntamente com o hipofluxo sistêmico, acarreta diminuição dos níveis de bicarbonato, instalando-se também a acidose metabólica ${ }^{(3,13)}$

Já no exame radiológico, verifica-se aumento progressivo da área cardíaca, com acentuação da trama vascular pulmonar. Nos casos em que o shunt E-D é pequeno, o exame radiológico pode ser normal ${ }^{(8)}$.

Nos casos de shunt E-D pequeno, o eletrocardiograma pode ser normal, porém, em situações de shunt com

Quadro 1 - Principais manifestações clínicas da PCA

\begin{tabular}{|l|l|}
\hline \multicolumn{1}{|c|}{ Comprometimento cardiocirculatório } & \multicolumn{1}{|c|}{ Comprometimento pulmonar } \\
\hline - Taquicardia, podendo estar presente o ritmo de galope & - Taquipnéia \\
- Sopro cardíaco, classicamente descrito como sopro & • Sinais de desconforto respiratório, como gemido \\
em maquinária & expiratório, retrações intercostais e subdiafragmáticas, \\
- Precórdio hiperdinâmico, caracterizado por ictus & - Episódios de apnéia decorrentes do estímulo dos \\
palpável ou visível & receptores J vagais por acúmulo de líquido pulmonar \\
- Aumento da amplitude dos pulsos & • Hipercapnia, havendo necessidade de suporte \\
- Aumento do diferencial pressórico, superior a & ventilatório \\
35mmHg, devido predominantemente à queda na & \\
pressão diastólica & \\
- Hepatomegalia, sinal tardio, secundário a ICC & \\
\hline
\end{tabular}


comprometimento hemodinâmico, observa-se desvio do eixo QRS para a esquerda e sinais de sobrecarga atrial e ventricular ${ }^{(2,8)}$.

O ecocardiograma possibilita a visualização direta do canal e de seu fluxo sangüíneo para a artéria pulmonar. Observa-se um estado de dilatação das câmaras esquerdas, em decorrência de hiperfluxo pulmonar, e maior retorno venoso pulmonar para o átrio esquerdo, o que justifica as relações átrio esquerdo/aorta superiores a $1,3^{(7,8,11,12,14)}$.

\section{Tratamento da PCA}

O tratamento clínico visa diminuir o hiperfluxo pulmonar e a sobrecarga hídrica, podendo-se utilizar restrição hídrica, diuréticos, drogas inotrópicas e outras medidas terapêuticas específicas $^{(1,3,8,12)}$.

Dentre as terapêuticas de suporte, está o uso de ventilação por pressão positiva, de forma invasiva ou não, com o objetivo de reduzir a resistência das vias aéreas e melhorar a complacência pulmonar, pois, a pressão positiva nas vias aéreas impede o colapso pulmonar, o que leva ao aumento da capacidade residual funcional, melhora da oxigenação, diminuição da resistência vascular pulmonar e redução do shunt e, portanto, do edema ${ }^{(1,3,7,15)}$. Além disso, devido ao estímulo dos receptores J vagais desencadeado pelo edema pulmonar, podem ocorrer episódios de apnéia, o que justifica o uso da pressão positiva ${ }^{(1,3,13)}$.

As drogas utilizadas para o fechamento do canal arterial são a indometacina e o ibuprofeno, sendo ambas eficazes, embora o ibuprofeno tenha sido estudado mais recentemen$\mathrm{te}^{(16)}$. Essas drogas são inibidoras da prostaglandina sintetase, usadas para prevenir ou reduzir a patência do canal arterial, sendo recomendadas em prematuros com idade gestacional $<34$ semanas nos primeiros 14 dias de vida. $O$ sucesso no fechamento do canal está presente em 60 a $80 \%$ dos casos, com redução da necessidade de intervenção cirúrgica e da incidência de displasia broncopulmonar (DBP) $)^{(1-3,7,16)}$

O tratamento cirúrgico está indicado quando há falha da terapêutica clínica após a administração da indometacina ou ibuprofeno ou quando existem contra-indicações para seu uso ${ }^{(1-3,7)}$. O procedimento cirúrgico mais utilizado é a clipagem do canal arterial por meio de toracotomia póstero-lateral, apresentando bons resultados e mortalidade inferior a $3 \% \%^{(1,3,15,17)}$.

Os cuidados pós-operatórios incluem a retirada do dreno de tórax nos primeiros dias, nos casos em que houve dissecção da pleura durante o procedimento cirúrgico, além da rigorosa vigilância com monitorização cardiorrespiratória e suporte clínico ${ }^{(3,15,17)}$.

\section{Complicações pulmonares na PCA}

A maioria dos defeitos congênitos cardíacos pode provocar alterações hemodinâmicas com repercussão no aparelho respiratório ${ }^{(18)}$. As principais complicações pulmonares da PCA em RNPT são atelectasias, infecções pulmonares e falha na extubação, que podem evoluir com deterioração aguda ou crônica da função pulmonar ${ }^{(3-6,9)}$.

A atelectasia é apontada como uma das principais causas de insuficiência respiratória. Sua incidência varia de 54 a 92\% nos neonatos, que são mais sensíveis ao efeito cumulativo de secreções pulmonares ${ }^{(18)}$. Tal característica decorre de particularidades da sua anatomia pulmonar, como pulmão imaturo com menor número de alvéolos, ventilação colateral limitada e diafragma também imaturo, predispondo à respiração desorganizada, com predomínio costal ${ }^{(5,6)}$. Além disso, quando o recém-nascido é submetido à cirurgia, após a realização da toracotomia, há o desaparecimento da pressão negativa intrapleural responsável pela manutenção dos pulmões inflados, levando ao colapso alveolar do pulmão homolateral à incisão cirúrgica. A toracotomia também promove alteração na integridade da parede torácica e na biomecânica dos músculos respiratórios, comprometendo a efetividade do trabalho respiratório ${ }^{(12,15,17-19)}$. A utilização de anestésicos durante a intervenção cirúrgica contribui para a formação de processos atelectásicos, pois promove redução da capacidade residual funcional, alteração da relação ventilação/perfusão e diminuição da função ciliar, favorecendo o desenvolvimento de infecções respiratórias ${ }^{(12,15,18,19)}$.

$\mathrm{O}$ aumento das prostaglandinas e a ativação de outros mediadores inflamatórios durante o processo infeccioso facilitam a patência ou reabertura tardia do canal, principalmente em recém-nascidos menores de $1.000 \mathrm{~g}^{(5)}$. As infecções pulmonares podem ocorrer em 9 a $21 \%$ dos neonatos submetidos à correção cirúrgica, aparecendo em mais de $50 \%$ daqueles submetidos à intubação traqueal, pois há tendência de colonização da traquéia e brônquios, aumentando para $28 \%$ a incidência de pneumonia neonatal, com mortalidade variando de 55 a $71 \%{ }^{(3,18,19)}$. O comprometimento pulmonar decorrente do PCA leva à necessidade de maior suporte ventilatório, com pressões mais elevadas e concentrações maiores de $\mathrm{O}_{2}$, além de maior período de ventilação mecânica, o que pode explicar a associação entre PCA e 
infecções, com risco aumentado de desenvolvimento de DBP e enfisema intersticial ${ }^{(3,5,9,10)}$.

Somado ao exposto acima, a desnutrição, muito freqüente nesses casos, é outro fator agravante, por comprometer a função respiratória, podendo levar à fadiga muscular e conseqüente insuficiência respiratória ${ }^{(20)}$.

\section{Intervenção fisioterapêutica}

De forma geral, a fisioterapia pode ser aplicada em recémnascidos com PCA, tanto na fase pré e pós-operatória, como naqueles que não necessitam de tratamento cirúrgico ${ }^{(18,19,21,22)}$.

O tratamento fisioterapêutico visa melhorar as condições pulmonares, aumentando a complacência pulmonar e diminuindo a resistência das vias aéreas e, dessa forma, reduz o trabalho respiratório imposto a esses neonatos. No pósoperatório, a fisioterapia objetiva otimizar o mecanismo de depuração mucociliar para facilitar a mecânica respiratória, além de prevenir e corrigir as complicações decorrentes do acúmulo de secreção pulmonar, como atelectasias, infecções e alterações da relação ventilação/perfusão, facilitando a extubação precoce ${ }^{(6,18,22-25)}$.

Dentre as técnicas e medidas fisioterapêuticas que podem ser empregadas nos neonatos sem necessidade de tratamento cirúrgico ou em fase pré-operatória, podem ser citados:

\section{Posicionamento}

O posicionamento adequado melhora a função pulmonar, favorece o desenvolvimento neurossensorial e psicomotor, além de propiciar maior conforto ao neonato ${ }^{(6,26)}$. A posição prona é relatada na literatura como uma boa alternativa para melhorar a oxigenação e a ventilação/perfusão, pois promove redistribuição da ventilação alveolar e da perfusão, aumento da complacência pulmonar e da capacidade residual funcional, melhora da função diafragmática e da ventilação dorsal pulmonar, bem como diminuição da assincronia toracoabdominal ${ }^{(6,18,26)}$.

O decúbito lateral tem sido utilizado em situações de atelectasias no pós-operatório de cirurgias cardíacas, para que a ventilação seja direcionada preferencialmente para o pulmão não-dependente, favorecendo à expansibilidade do pulmão atelectásico ${ }^{(6,23,26)}$. Entretanto, a alternância periódica dos decúbitos deve ser realizada, para não haver hipoventilação em outras áreas pulmonares, sempre se respeitando as limitações do neonato - em especial com o lado homolateral à incisão cirúrgica pela possível presença de dor e do dreno torácico nos primeiros dias após a cirurgia ${ }^{(6)}$.

\section{Reequilíbrio toracoabdominal}

Outra técnica que pode ser empregada é o método do reequilíbrio toracoabdominal (RTA). Este objetiva recuperar o sinergismo entre o tórax e o abdome, melhorando a justaposição entre o diafragma e as costelas e aumentando o tônus e a força dos músculos respiratórios. Desta forma, os músculos respiratório podem desempenhar com eficiência suas funções inspiratórias e expiratórias, com aumento do volume corrente, melhora da complacência pulmonar e diminuição da resistência expiratória em RNPT ${ }^{(27)}$. Dentre as manobras específicas do RTA que podem ser empregadas, destacam-se ${ }^{(27)}$ :

- Apoio toracoabdominal: melhora o componente justaposicional do diafragma. O terapeuta deve colocar uma das mãos sobre a região inferior do tórax e superior do abdome e parte dos seus dedos deve alcançar as costelas, o que permitirá o direcionamento das costelas para a posição normal. Tentar, na expiração, tracionar suavemente as costelas para baixo e manter nessa posição na inspiração, já que a freqüência respiratória do recém-nascido é muito alta. Como resultado da técnica, deve-se observar aumento do movimento no abdome superior durante a inspiração, o que reflete a melhora na complacência e na ventilação pulmonar.

- Apoio abdominal inferior: também objetiva melhorar o componente justaposicional. O fisioterapeuta deve aplicar pressão no abdome inferior, durante a inspiração. A pressão aplicada deve ser suficiente para ser vencida pelo diafragma do neonato e não aumentar o uso dos músculos acessórios da inspiração. Como resultado, deve-se esperar um movimento inspiratório positivo por todo o abdome e discreta elevação das seis últimas costelas e da região inferior do esterno.

- A longamento posterior: consiste em facilitar a ação diafragmática e as reações de retificação e equilíbrio, além de proporcionar o posicionamento correto da caixa torácica. Uma das mãos do fisioterapeuta deve estar posicionada na região occipital e a outra, na região sacral, para realizar leve alongamento de tronco no sentido crânio-caudal na expiração e manter na inspiração.

\section{Técnicas de higiene brônquica}

As manobras de higiene visam auxiliar a depuração das vias aéreas por meio da drenagem postural e/ou da vibração torácica manual, enquanto a aspiração traqueal tenta substituir a tosse $\mathrm{e}^{(6,23,25)}$.

Estudos demonstram aumento nos níveis de $\mathrm{PaO}_{2}$, melhora na ausculta pulmonar e na perfusão periférica em 
RNPT em ventilação mecânica invasiva com insuficiência respiratória, quando se realizam manobras de higiene brônquica $^{(22,24,25)}$. Além disso, revisões sistemáticas demonstraram que tais manobras no período periextubação diminuem a necessidade de reintubações e reduzem os episódios de atelectasias pós-extubação ${ }^{(22,28)}$.

Já as técnicas que visam aumentar o fluxo expiratório são baseadas na fisiologia da tosse, cujo objetivo principal é o de gerar maior velocidade de fluxo de gás na fase expiratória, com o intuito de desprender as secreções aderidas na árvore brônquica ${ }^{(6,18,23)}$. Tais técnicas podem ser aplicadas em neonatos submetidos à correção da PCA, hemodinamicamente estáveis. Entretanto, deve-se verificar e avaliar a presença de desconforto e/ou dor no pós-operatório, identificando o momento propício para aplicar tais técnicas.

Nos prematuros com idade gestacional $<34$ semanas e/ou $<1.500 \mathrm{~g}$, principalmente nas primeiras 72 horas de vida, as técnicas de higiene brônquica, como drenagem postural, vibração torácica manual, aumento de fluxo expiratório e aspiração, devem ser realizadas com cautela, devido ao maior risco de hemorragia periintraventricular pela imaturidade vascular do sistema nervoso central. Além disso, as manobras compressivas são evitadas, pois podem ocasionar colabamento das vias aéreas e diminuição do volume pulmonar, resultando em atelectasias ${ }^{(6)}$.

A manobra de bag squeezing modificada promove aumento do fluxo expiratório. É utilizada em neonatos intubados que cursam com quadro pulmonar secretivo. A técnica consiste na insuflação dos pulmões com balão auto-inflável até uma pressão de cerca de $5 \mathrm{cmH}_{2} \mathrm{O}$ acima do pico de pressão inspiratória utilizada durante a ventilação pulmonar mecânica. Ao final da inspiração manual, o balão auto-inflável deve ser liberado subitamente e iniciada, simultaneamente, a vibração manual da caixa torácica até o final da expiração ${ }^{(6,18)}$. Entretanto, em decorrência das limitações impostas pela cirurgia, deve-se aplicar o aumento do fluxo expiratório somente com balão auto-inflável que, ao contrário do bag squeenzing modificado, não utiliza vibrações torácicas associadas com a insuflação ${ }^{(6)}$.

\section{Técnicas de reexpansão pulmonar}

Tais técnicas são importantes no pós-operatório, uma vez que, nessas circunstâncias, os neonatos tendem a perder volumes e capacidades pulmonares ${ }^{(21,23,25)}$. Na manobra de insuflação pulmonar manual, o neonato é desconectado da ventilação mecânica e seus pulmões são insuflados com o balão auto-inflável, mantendo-se em torno de dois a três segundos cada insuflação. É indicada para prevenir atelectasias, reexpandir áreas colapsadas e minimizar risco de hipoxemia ${ }^{(23)}$.

Outra técnica é o direcionamento de fluxo, com a qual a mão do terapeuta exerce uma pressão (bloqueio) no hemitórax contra-lateral à incisão cirúrgica, buscando direcionar o fluxo a fim de expandir o pulmão contra-lateral ${ }^{(23)}$.

\section{CPAP Nasal}

O CPAP também é um recurso para os neonatos submetidos à correção cirúrgica de PCA. Devido ao seu efeito estabilizador das vias aéreas, da caixa torácica e do volume pulmonar, o uso do CPAP tem sido a estratégia ventilatória preferida para auxiliar no processo de retirada da ventilação pulmonar mecânica, em particular no neonato de muito baixo peso. Evidências recentes mostram que o CPAP, em especial quando aplicado por meio da pronga nasal, reduz a incidência de eventos adversos, como atelectasias pós-extubação, episódios de apnéia, acidose respiratória e necessidade de reintubação traqueal, além de diminuir a freqüência de recém-nascidos dependentes de oxigênio aos 28 dias de vida ${ }^{(1,29)}$.

\section{Considerações finais}

As principais complicações pulmonares em recém-nascidos com PCA são atelectasias, infecções pulmonares e falha na extubação, que levam ao aumento do tempo de ventilação mecânica e de internação hospitalar. Em conseqüência, há maior risco de novas complicações, como infecções de repetição, DBP e aumento na mortalidade.

Em longo prazo, o prognóstico da PCA está associado à presença de complicações respiratórias, destacando-se, sobretudo, nos primeiros anos de vida, as infecções pulmonares de repetição, os quadros de broncoespasmo e o deficit de crescimento pôndero-estatural.

Dessa forma, a fisioterapia respiratória contribui de forma importante na evolução de recém-nascidos com tais complicações. Entretanto, na literatura, ainda existem poucos estudos mostrando as técnicas fisioterapêuticas com suas vantagens e desvantagens, sendo necessárias novas pesquisas sobre técnicas específicas em recém-nascidos. 


\section{Referências bibliográficas}

1. Barros MCM. Persistência do canal arterial. In: Kolpelman BI, Santos AMN, Goulart AL, Almeida MFB, Miyoshi MH, Guinsburg R, editores. Diagnóstico e tratamento em neonatologia. São Paulo: Atheneu; 2004. p. 259-65.

2. Laughon MM, Simmons MA, Bose CL. Patency of the ductus arteriosus in the premature infant: is it pathologic? Should it be treated? Curr Opin Pediatr 2004;16:146-51.

3. Hermes-DeSantis ER, Clyman RI. Patent ductus arteriosus: pathophysiology and management. J Perinatol 2006;26 (Suppl 1):S14-8.

4. Miyague NI. Preterm neonates with patent ductus arteriosus. J Pediatr (Rio J) 2005;81:429-30.

5. Gonzalez A, Sosenko IR, Chandar J, Hummler H, Claure N, Bancalari E. Influence of infection on patent ductus arteriosus and chronic lung disease in premature infants weighing 1000 grams or less. J Pediatr 1996;128:470-8

6. Maccari GM, Abreu CF, Miyoshi MH. Fisioterapia respiratória nas doenças respiratórias neonatais. In: Kolpelman BI, Santos AMN, Goulart AL, Almeida MFB, Miyoshi MH, Guinsburg R, editores. Diagnóstico e tratamento em neonatologia. São Paulo: Atheneu; 1998. p. 213-26.

7. Haro FMB, Sharovsky R, Pereira AMAG, Costa MTZ, Okay Y, Vaz FAC. Persistência do canal arterial no período neonatal. Pediatria (São Paulo) 1997;19:267-79.

8. Carvalho WB, Carvalho MF. Persistência do canal arterial. In: Fazio Jr J, Carvalho MF, Nogueira PRC, Carvalho WB, editores. Cuidados intensivos no período neonatal. São Paulo: Sarvier; 1999. p. 94-6.

9. Koch J, Hensley G, Roy L, Brown S, Ramaciotti C, Rosenfeld CR. Prevalence of spontaneous closure of the ductus arteriosus in neonates at a birth weight of 1000 grams or less. Pediatrics 2006;117:1113-21.

10. Walther FJ, Benders MJ, Leighton JO. Early changes in the neonatal circulatory transition. J Pediatr 1993;123:625-32.

11. Su BH, Watanabe T, Shimizu M, Yanagisawa M. Doppler assessment of pulmonary artery pressure in neonates at risk of chronic lung disease. Arch Dis Child Fetal Neonatal Ed 1997;77:23-7.

12. Jacob JLB, Braile DM. Current treatment of the persistent arterial duct. Rev Bras Cir Cardiovasc 2003;18:350-8.

13. El Hajjar M, Vaksmann G, Rakza T, Kongolo G, Storme L. Severity of the ductal shunt: a comparison of different markers. Arch Dis Child Fetal Neonatal Ed 2005;90:419-22.

14. Afiune JY, Singer JM, Leone CR. Echocardiographic post-neonatal progress of preterm neonates with patent ductus arteriosus. J Pediatr (Rio J) 2005;81:454-60.

15. Stayer SA, Diaz LK, East DL, Gouvion JN, Vencill TL, McKenzie ED et al. Changes in respiratory mechanics among infants undergoing heart surgery. Anesth Analg 2004;98:49-55.
16. Ohlsson A, Walia R, Shah S. Ibuprofen for the treatment of patent ductus arteriosus in preterm and/or low birth weight infants. Cochrane Database Syst Rev 2003;(2):CD003481.

17. McCurnin DC, Yoder BA, Coalson J, Grubb P, Kerecman J, Kupferschmid J et al. Effect of ductus ligation on cardiopulmonary function in premature baboons. Am J Respir Crit Care Med 2005;172:1569-74.

18. Leginestra M, Amorin EF. Atendimento fisioterapêutico em pós-operatório de cirurgia cardíaca em crianças. In: Regenga MM, editor. Fisioterapia em cardiologia: da unidade de terapia intensiva à reabilitação. São Paulo: Roca; 2000. p. 169-98.

19. Brown KL, Ridout DA, Goldman AP, Hoskote A, Penny DJ. Risk factors for long intensive care unit stay after cardiopulmonary bypass in children. Crit Care Med 2003;31:28-33.

20. Mota EM, Garcia PCR, Piva JP, Fritscher CC. The influence of poor nutrition on the necessity of mechanical ventilation among children admitted to the Pediatric Intensive Care Unit. J Pediatr (Rio J) 2002;78:146-52.

21. Hussey J. Effects of chest physiotherapy for children in intensive care after surgery. Physioterapy 1992;78:109-13.

22. Flenady VJ, Gray PH. Chest physiotherapy for preventing morbidity in babies being extubated from mechanical ventilation. Cochrane Database Syst Rev 2000;(2):CD000283.

23. Presto $B$, Presto LDN. Técnicas e manobras fisioterapêuticas. In: Presto $B$, Presto LDN, editores. Fisioterapia respiratória: uma nova visão. Rio de Janeiro: Bruno Presto; 2003. p. 97-128.

24. Krause MF, Hoehn T. Chest physiotherapy in mechanically ventilated children: a review. Crit Care Med 2000;28:1648-51.

25. Lewis JA, Lacey JL, Henderson-Smart DJ. A review of chest physiotherapy in neonatal intensive care units in Australia. J Paediatr Child Health 1992;28:297-300.

26. Antunes LCO, Rugolo LMSS, Crocci AJ. Efeito da posição do prematuro no desmame da ventilação mecânica. Rev Chil Pediatr 2005;76:436-7.

27. Zanchet RC, Chagas AM, Melo JS, Watanabe PY, Simões-Barbosa A, Feijó G. Influence of the technique of re-educating thoracic and abdominal muscles on respiratory muscle strength in patients with cystic fibrosis. J Bras Pneumol 2006;32:123-9.

28. Halliday HL. What interventions facilitate weaning from the ventilator? A review of the evidence from systematic reviews. Paediatr Respir Rev 2004;5 (Suppl A); S347-52.

29. Ammari A, Suri M, Milisavljevic V, Sahni R, Bateman D, Sanocka U et al. Variables associated with the early failure of nasal CPAP in very low birth weight infants. J Pediatr 2005;147:341-7. 